\title{
OPEN Infant's Behaviour Checklist for low birth weight infants and later neurodevelopmental outcome
}

\author{
Hideki Kihara ${ }^{1}$, Hisako Nakano ${ }^{2}$, Tomohiko Nakamura ${ }^{3} \&$ Hirotaka Gima ${ }^{4,5}$
}

Assessment of the characteristics of spontaneous movements and behaviour in early infancy helps in estimating developmental outcomes. We introduced the Infant Behaviour Checklist (IBC) and examined the relationship between the behavioural characteristics of low-birth-weight infants and neurodevelopmental outcomes at 6 years of age. The behavioural characteristics during the neonatal (36-43 weeks, adjusted) and early infancy periods (49-60 weeks, adjusted) were assessed in verylow-birth-weight infants. The IBC includes 44 common behaviours. We assessed the appearance of individual behavioural characteristics at each period according to the neurodevelopmental outcome. Of the 143 infants assessed during the neonatal period, 89 had typical development (TD), 30 had intellectual disability (ID), and 24 had autism spectrum disorder (ASD). In 78 infants assessed during early infancy, 40, 21, and 17 had TD, ID, and ASD, respectively. The frequency of appearance of three behaviour-related items was significantly lower in the ID group than in the TD group. The frequency of appearance of three posture- and behaviour-related items was significantly lower, while that of two posture-related items was significantly higher, in the ASD group than in the TD group. Behavioural assessment using the IBC may provide promising clues when considering early intervention for lowbirth-weight infants.

Developments in medical technology have markedly improved the survival rates of low-birth-weight infants; however, such infants have increased risks of neurodevelopmental abnormalities ${ }^{1,2}$. Several studies have also reported that preterm birth and low-birth-weight are risk factors for future developmental disabilities such as intellectual disability (ID) ${ }^{3,4}$ and autism spectrum disorder (ASD) ${ }^{5-9}$. In recent years, assessing neurological development based on the characteristics of spontaneous movements and behaviours can be useful in considering interventions for infants at risk of developmental problems. In particular, it has been reported that a type of spontaneous movement, defined by Prechtl as general movements $(\mathrm{GMs})^{10}$, is an early sensitive marker of cerebral palsy (CP) later in life $\mathrm{e}^{11,12}$. Furthermore, a relationship between the qualitative abnormality of GMs, $\mathrm{ID}^{13,14}$, and $\mathrm{ASD}^{15-17}$ has been reported, but its accuracy is still inadequate compared with that of the relationship with CP. Regarding ID and ASD, observing the various behavioural characteristics rather than the qualitative abnormalities of GMs may be useful as an early sign ${ }^{18-26}$.

We have focused on the characteristics of spontaneous movements in early infancy and reported their relationship with neurological development ${ }^{26-29}$. We have reported that it is useful to quantify the various spontaneous movements that infants show; however, a detailed evaluation of their behavioural characteristics has thus far been sufficient. Therefore, we conducted a detailed observational evaluation of behavioural characteristics during early infancy in low-birth-weight infants using videos recorded for the assessment of GMs. In this study, we introduced the Infant's Behaviour Checklist (IBC) and examined the relationship between behavioural characteristics of low-birth-weight infants assessed using the IBC and their neurodevelopmental outcomes at 6 years of age. The IBC lists 44 items considered important in assessing spontaneous movement and behavioural and postural characteristics of low-birth-weight infants. Clarifying the relationship between detailed behavioural characteristics in early infancy and later neurological development is important for enhancing developmental care for low-birth-weight infants.

\footnotetext{
${ }^{1}$ Babycastle Corporation, 373-1, Tsubuku-imamachi, Kurume City, Fukuoka 830-8630, Japan. ${ }^{2}$ Department of Physical Therapy, Kyorin University, 5-4-1 Shimorenjaku, Mitaka City, , Tokyo 181-8612, Japan. ${ }^{3}$ Department of Neonatology, Nagano Children's Hospital, 3100, Toyoshina, Azumino City, Nagano 399-8288, Japan. ${ }^{4}$ Department of Physical Therapy, Faculty of Health Sciences, Tokyo Metropolitan University, 7-2-10 Higashi-Ogu, Arakawa-ku, Tokyo 116-8551, Japan. ${ }^{5}$ Department of Physical Therapy, Graduate School of Human Health Sciences, Tokyo Metropolitan University, 7-2-10 Higashi-Ogu, Arakawa-ku, Tokyo 116-8551, Japan. ${ }^{\circledR}$ email: gima@tmu.ac.jp
} 


\begin{tabular}{|l|l|l|l|}
\hline & TD & ID & ASD \\
\hline Number & 89 & 30 & 24 \\
\hline Male/female (n) & $30 / 59$ & $17 / 12$ & $14 / 10$ \\
\hline Birthweight (g), median (range) & $1,058(489-1495)$ & $931(460-1498)$ & $827^{*}(588-1346)$ \\
\hline Gestational age (weeks and days), median (range) & $28 w 5 d$ (22w5d-34w4d) & $27 w 2 d(23 w 0 d-36 w 3 d)$ & $27 w 2 d(23 w 6 d-33 w 0 d)$ \\
\hline $\begin{array}{l}\text { Age at recording of behavioural development (weeks and } \\
\text { days), median (range) }\end{array}$ & $38 w 0 d$ (36w0d-43w2d) & $39 w 2 d$ (36w1d-43w4d) & $39 w 5 d(36 w 4 d-43 w 1 d)$ \\
\hline
\end{tabular}

Table 1. Characteristics and developmental outcome of the neonatal assessment group. TD typical development, $I D$ intellectual disability, $A S D$ autism spectrum disorder. ${ }^{*} p<0.05$ in comparison with TD group using the Mann-Whitney U test.

\begin{tabular}{|l|l|l|l|}
\hline & TD & ID & ASD \\
\hline Number & 40 & 21 & 17 \\
\hline Male/female (n) & $13 / 27$ & $7 / 14$ & $9 / 8$ \\
\hline Birthweight (g), median (range) & $983(492-1468)$ & $790(502-1498)$ & $849(546-1414)$ \\
\hline Gestational age (weeks and days), median (range) & $28 w 6 d(23 w 2 d-32 w 4 d)$ & $27 w 0 d(23 w 0 d-36 w 3 d)$ & $27 w 2 d(23 w 6 d-33 w 0 d)$ \\
\hline $\begin{array}{l}\text { Age at recording of behavioural development (weeks and } \\
\text { days), median (range) }\end{array}$ & $54 w 0 d(49 w 0 d-58 w 1 d)$ & $53 w 1 d(49 w 6 d-59 w 0 d)$ & $53 w 3 d(50 w 0 d-60 w 2 d)$ \\
\hline
\end{tabular}

Table 2. Characteristics and developmental outcome of the early infancy assessment group. TD typical development, ID intellectual disability, $A S D$ autism spectrum disorder. ${ }^{\star} p<0.05$ in comparison with TD group using the Mann-Whitney U test.

\section{Results}

Participant characteristics. The distribution of the developmental outcomes at age 6 years in neonatal assessment (NA) was as follows: typical development (TD), $n=89 ; \mathrm{ID}, \mathrm{n}=30$; and ASD, $\mathrm{n}=24$. The distribution of the developmental outcomes at age 6 years in early infancy assessment (EA) was as follows: TD, $n=40$; ID, $\mathrm{n}=21$; and ASD, $\mathrm{n}=17$. For the 45 infants who belonged to both groups, the distribution of the developmental outcomes at age 6 years was as follows: TD, $n=25 ; \mathrm{ID}, \mathrm{n}=11$; and ASD, $\mathrm{n}=9$.

The background factors of NA and EA are listed in Tables 1 and 2, respectively. In the NA group, the birth weight was significantly lower in the ASD than in the TD group. In the EA group, there were no significant differences between the groups in terms of birth weight, gestational age (weeks), or age in weeks at video recording.

Evaluation of the behavioural characteristics using IBC. The frequency of observation of each behaviour in all groups for NA and EA is shown in Table 3. In the NA group, the ID group exhibited significantly fewer instances of trunk rotation (item 19,10\%), separate finger flexion and extension (item 25, 20\%), and lower limb flexion and hip internal rotation and external rotation (item 27, 27\%) than did the TD group. In contrast, there were no items in the ASD group that showed a significant difference. In the EA group, the ID group showed significantly fewer instances of toe flexion and extension separately (item 32, 19\%) than did the TD group. Meanwhile, the ASD group showed significantly more instances of asymmetrical tonic neck reflex (ATNR) (item 3, $35 \%$ ) and toe flexion (item $16,41 \%$ ), and there were significantly fewer instances of head at the midline (item 2 , $0 \%$ ), neck rotation (item $18,29 \%$ ), and wrist rotation (item $24,0 \%$ ).

Figure 1 shows the distribution of the data for each item in the ID and the ASD groups at NA and EA terms by the difference of percentage of participants for whom the posture and behaviour appeared. Each line represents the value of the TD group minus the value of the ID (blue line) and the ASD (red line) group, with larger positive values indicating a higher proportion of appearance compared to the TD group, and larger negative values indicating a lower proportion of appearance.

\section{Discussion}

In this study, we introduced the IBC and examined the relationship between the behavioural characteristics of low-birth-weight infants assessed using IBC and their neurodevelopmental outcomes at 6 years of age. In particular, we focused on ID and ASD as developmental outcomes, and examined the characteristics of spontaneous movements and behaviour during the neonatal and early infancy periods. These periods are considered to be important for qualitative evaluation of the characteristics of spontaneous movements ${ }^{10-12,30}$ and also from the viewpoint of the developmental mechanism of the central nervous system ${ }^{10,31-33}$. In particular, fidgety movements (FMs) which appear during the EA period, have been reported to be strongly related to later neurological developmental outcomes ${ }^{10-17,29-33}$. The assessment of characteristics of spontaneous movements and behaviour at both NA and EA is considered important to verify early motor signs of ID and ASD.

Evaluation using the IBC revealed the following behavioural characteristics in the ID group: (1) Among the items of IBC, a significantly lower frequency of appearance was found in some items related to behaviour, but not in items related to posture. (2) The number of items related to behaviour with a significantly lower frequency 


\begin{tabular}{|c|c|c|c|c|c|c|}
\hline \multirow[b]{2}{*}{ Item number } & \multicolumn{3}{|c|}{ Neonatal assessment group } & \multicolumn{3}{|c|}{ Early infancy assessment group } \\
\hline & TD $(n=89)$ & ID $(n=30)$ & $\operatorname{ASD}(n=24)$ & TD $(n=40)$ & ID $(n=21)$ & $\operatorname{ASD}(n=17)$ \\
\hline 1 & 2 & 3 & 4 & 8 & 19 & 18 \\
\hline 2 & - & - & - & 33 & 10 & $0^{*}$ \\
\hline 3 & 3 & 0 & 4 & 5 & 14 & $35^{*}$ \\
\hline 4 & 1 & 0 & 4 & 0 & 5 & 6 \\
\hline 5 & 1 & 7 & 0 & 0 & 10 & 0 \\
\hline 6 & 39 & 60 & 50 & 28 & 24 & 35 \\
\hline 7 & 18 & 10 & 13 & 50 & 43 & 24 \\
\hline 8 & 6 & 10 & 4 & 5 & 10 & 6 \\
\hline 9 & 20 & 23 & 50 & 3 & 0 & 24 \\
\hline 10 & 7 & 7 & 4 & 8 & 0 & 0 \\
\hline 11 & 6 & 3 & 4 & 3 & 0 & 0 \\
\hline 12 & 20 & 17 & 13 & 20 & 10 & 0 \\
\hline 13 & - & - & - & \begin{tabular}{|l|}
78 \\
\end{tabular} & 62 & 59 \\
\hline 14 & 10 & 10 & 13 & 0 & 0 & 0 \\
\hline 15 & 7 & 13 & 17 & 3 & 5 & 6 \\
\hline 16 & - & - & - & 8 & 24 & $41^{*}$ \\
\hline 17 & 10 & \begin{tabular}{|l|l|}
10 \\
\end{tabular} & 8 & 8 & 0 & 0 \\
\hline 18 & 30 & 10 & 30 & 75 & 43 & $29^{*}$ \\
\hline 19 & 44 & $10^{*}$ & 21 & 25 & 24 & 18 \\
\hline 20 & 6 & 10 & 4 & 0 & 0 & 6 \\
\hline 21 & 76 & 80 & 54 & \begin{tabular}{|l|}
73 \\
\end{tabular} & 52 & 47 \\
\hline 22 & 18 & 7 & 4 & 65 & 43 & 47 \\
\hline 23 & 7 & 13 & 4 & 0 & 5 & 0 \\
\hline 24 & - & - & - & 43 & 19 & $0^{*}$ \\
\hline 25 & 53 & $20^{*}$ & 17 & 38 & 29 & 35 \\
\hline 26 & 3 & 13 & 8 & 10 & 14 & 6 \\
\hline 27 & 56 & $27^{*}$ & 33 & 58 & 29 & 53 \\
\hline 28 & 1 & 3 & 4 & 18 & 0 & 0 \\
\hline 29 & 45 & 27 & 29 & 93 & 86 & 82 \\
\hline 30 & 16 & 0 & 17 & 40 & 19 & 12 \\
\hline 31 & - & - & - & 98 & 81 & 100 \\
\hline 32 & - & - & - & 63 & $19^{*}$ & 41 \\
\hline 33 & 8 & 0 & 8 & 25 & 24 & 24 \\
\hline 34 & 53 & \begin{tabular}{|l|}
27 \\
\end{tabular} & 29 & 35 & 19 & 41 \\
\hline 35 & 38 & 23 & 8 & 18 & 14 & 12 \\
\hline 36 & 55 & 43 & 33 & 5 & 10 & 12 \\
\hline 37 & 10 & 7 & 0 & 10 & 5 & 6 \\
\hline 38 & - & - & - & 3 & 5 & 6 \\
\hline 39 & 49 & 27 & 25 & 53 & 43 & 53 \\
\hline 40 & 74 & $\begin{array}{l}67 \\
\end{array}$ & $\begin{array}{l}79 \\
\end{array}$ & 0 & 0 & 12 \\
\hline 41 & 45 & \begin{tabular}{|l}
57 \\
\end{tabular} & 54 & 0 & 0 & 6 \\
\hline 42 & 8 & \begin{tabular}{|l|l}
17 \\
\end{tabular} & 13 & 8 & 0 & 6 \\
\hline 43 & 5 & 17 & 17 & 0 & 0 & 0 \\
\hline 44 & 1 & \begin{tabular}{|l|}
0 \\
\end{tabular} & 8 & 0 & 0 & 0 \\
\hline
\end{tabular}

Table 3. Percentage of presence behaviour in all items by group in the neonatal and early infancy assessment. TD typical development, ID intellectual disability, $A S D$ autism spectrum disorder. ${ }^{\star} p<0.05$ in comparison with TD group using the Fisher's exact test (corrected by the Benjamini-Hochberg method).

of appearance was larger in the NA group than that in the EA group. In the ID group, the frequencies of items 19 (trunk rotation), 25 (separate finger flexion and extension), and 27 (lower limb flexion and hip internal rotation and external rotation) were significantly lower in NA; in contrast, the frequency of item 32 (toe flexion 
Neonatal Assessment group

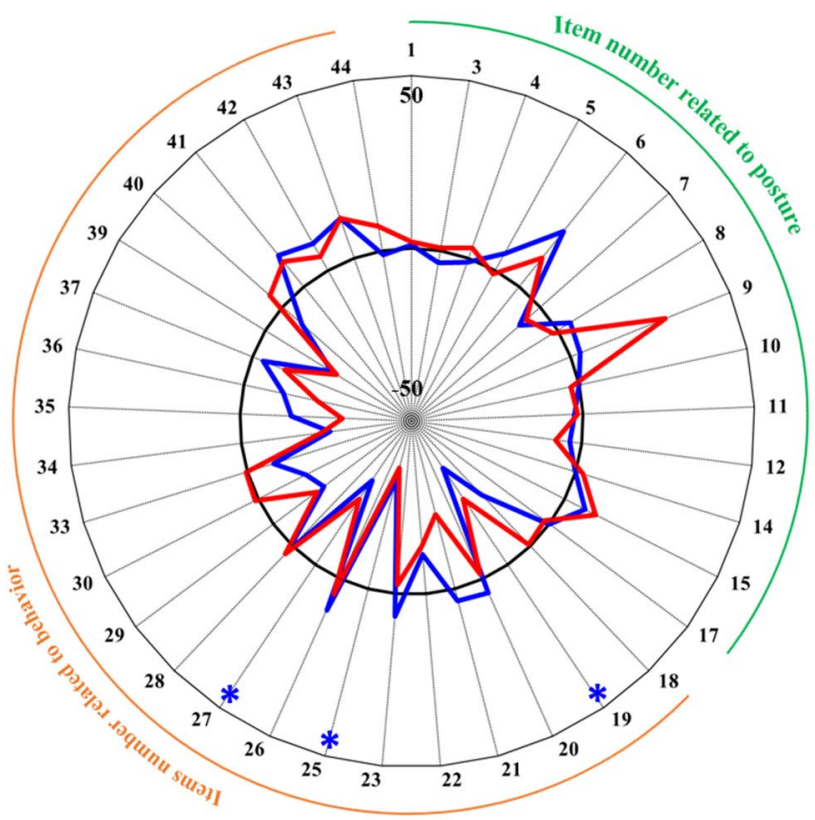

Early infancy Assessment group

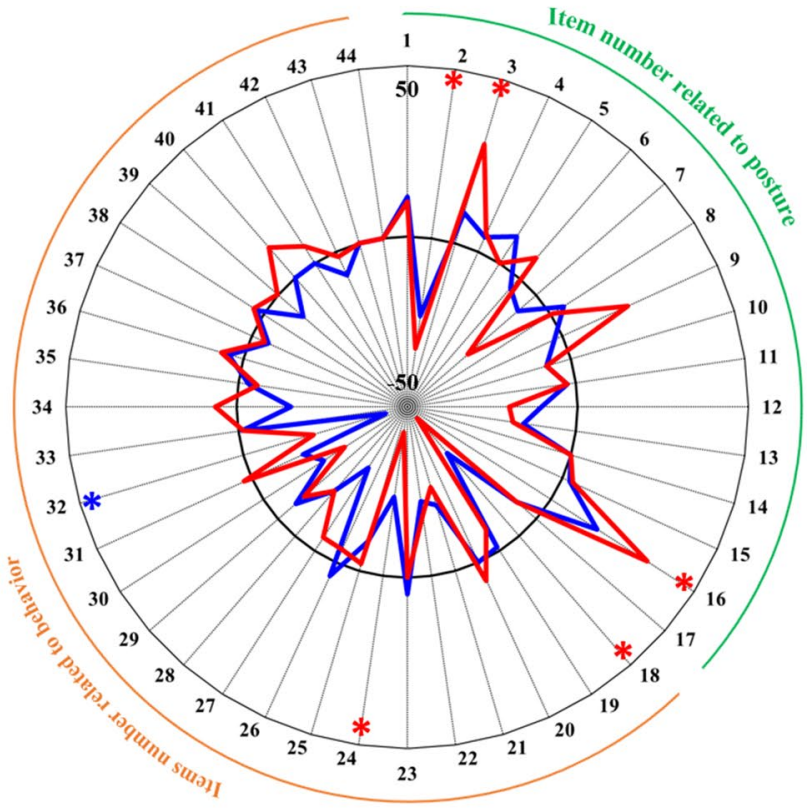

Typical development Intellectual disability

Autism spectrum disorder

Figure 1. Radar chart of the difference percentage between the typical development group and the other group at NA and EA terms. The red and blue line shows the distribution of the difference of percentage of participants for whom the posture and behaviour appeared. Each line represents the value of the TD group minus the value of the ID and the ASD group (note that the values of the TD group are all zero and the values have no units). ${ }^{*} p<0.05$ in comparison with the TD group using Fisher's exact test corrected by the Benjamini-Hochberg method.

and extension separately) was significantly lower in EA. Meanwhile, there were no items related to posture that showed a significant difference between NA and EA. The characteristic behaviour in the ID group may reflect the low quantitative characteristics of spontaneous movements and behaviour in the ID group. A previous study that examined the characteristics of spontaneous limb movements by computer-based analysis using the same image data as in this study reported that the value of the quantitative indices (average velocity and number of movement units per min) in the NA was significantly lower in the ID than in the TD group ${ }^{27}$. In the evaluation using the IBC, items related to behaviour were judged 'present' when the behaviour was observed at least twice. Since a reduction in the activity of spontaneous movements also leads to a reduced frequency of appearance of each behaviour, it is considered that a significantly lower frequency of appearance in items related to behaviour was observed in the ID group. However, a previous study examining the characteristics of spontaneous limb movement in EA reported no significant difference in the values of quantitative indices between the ID group and the TD group ${ }^{26}$. The quantitative characteristics of spontaneous movements in the ID group may be more apparent in the NA than in the EA group. The results of the present study also indicated that the quantitative characteristics of spontaneous movements and behaviour in the ID group may appear more prominently in the NA than in the EA group, suggesting the importance of evaluating NA for the ID group.

The important results in the evaluation of the ASD group were as follows: (1) There were no items in the NA group that showed a significant difference. (2) In the EA group, there were many items related to posture that showed a significant difference in the appearance rate. (3) All items that showed a significant difference in the appearance rate were related to the movement of the neck and distal part of the upper and lower limbs. Previous studies that examined early signs of ASD by assessing the characteristics of spontaneous movements, posture, and behaviour reported features, such as abnormal upper extremity muscle tone $e^{21}$, postural asymmetries during lying ${ }^{18,20}$, head-lag during the pull-to-sit transition ${ }^{23}$, oral-motor abnormalities ${ }^{22}$, motor developmental delay in early infancy ${ }^{24}$, qualitative abnormalities in $\mathrm{GMs}^{16,17}$, poorer performance in maintaining the midline position of the head ${ }^{26}$, and lower motion complexity in legs ${ }^{34}$. All these early signs of ASD have been observed after 3 months of age. Therefore, it is highly possible that the results of this study also contain more information on the early behavioural characteristics of ASD in EA than in NA. In the EA evaluation, the frequencies of items 2 (head midline), 18 (neck rotation), and 24 (wrist rotation) were significantly lower, and 3 (ATNR) and 16 (toe flexion) were significantly higher in the ASD than in the TD group. Of these, items related to head movement and position (head midline, neck rotation, and ATNR) were considered to reflect the poorer performance in maintaining the midline position of the head in the previous study ${ }^{26}$. Meanwhile, the small amount of wrist rotation and the 
high ratio toe flexion may reflect the abnormality of FMs in ASD. FMs are a type of GM characterized by small continual movements of moderate speed and variable acceleration of the neck, trunk, and limbs in all directions that appears in early infancy and is defined as 'an ongoing stream of small and circular movements of limbs ${ }^{32,33}$. A study examining the kinematic features of FMs reported that the value of curvature in the movement trajectory of the distal limbs was related to the frequency of FMs appearance ${ }^{35}$. This value of the curvature is the magnitude by which a trajectory deviates from being straight and reflects the fine rotational movement of the wrist and the ankle. Previous studies have reported that the appearance of FMs is often absent in infants with ASD ${ }^{15-17}$, and the characteristics of distal limb movements and posture in the ASD group in this study were considered to be related to FMs abnormalities (absence of FMs), similar to results of previous studies.

Preterm birth and low-birth-weight are risk factors for $\mathrm{ASD}^{5-9}$. However, detecting the early motor signs of ASD during early infancy is a considerable challenge. We used the IBC to investigate the relationship between behaviours in the neonatal and early infancy periods with development at the age of 6 years due to disability. We also ascertained the characteristics of behaviours by disability in the neonatal and early infancy periods. Behavioural characteristics during early infancy in low-birth-weight infants reflected the diagnosis of later ID and ASD. Identifying factors that allow the early recognition of ASD is crucial because early intervention results in the development of better socially engaged imitation, eye contact, cognitive ability, adaptive behaviour, socialization, daily living skills, and motor skills for $\mathrm{ASD}^{36,37}$. Behavioural assessment using the IBC may provide promising clues when considering early intervention for low-birth-weight infants.

The limitations of this study need to be considered. Firstly, for consistency, the observational assessments were all performed by one assessor. In clinical practice, it would be important to have more than three assessors to ensure a reliable evaluation. Secondly, our study's assessments using the IBC did not compare the severity of disabilities or scoring. Furthermore, several participants (13 of 24 in the NA group and 3 of 17 in the EA group) in the ASD group had difficulty in completing the Wechsler Intelligence Scale for Children (WISC) III due to the characteristics of ASD; thus, their intelligence quotient (IQ) could not be assessed accurately (see Supplementary Fig. S1 online). This is an important issue worthy of future investigation. Therefore, we would like to conduct further studies using a larger sample size to investigate the disability severity grading and scoring and the impact of comorbid ASD and ID.

\section{Methods}

Ethics. The present study was approved by the Institutional Review Board of Nagano Children's Hospital. The infants' parents or guardians were provided with both written and oral explanations of how the data would be handled (use in written reports and presentations at academic conferences, protection of private information) before they provided signed consent. All procedures were performed in accordance with the approved guidelines and regulations.

Participants. We included 564 extremely-low-birth-weight infants who were hospitalized in the neonatal intensive care unit of Nagano Children's Hospital between 2002 and 2010. We assessed the behaviours of 143 such infants during the neonatal period ( 36 weeks 0 days to 43 weeks 6 days, adjusted, the NA group) and 78 infants in the early infancy period ( 49 weeks 0 days to 60 weeks 6 days, adjusted, the EA group) (Fig. 2).

Recording of behaviour. The infants' behaviours were recorded by videography (DCR-TRV20 or DVRPC350, SONY, Tokyo, Japan). The video recording method was modelled on that of Einspieler et al..$^{23}$ The infants in the NA group were placed supine on a cot in the neonatal intensive care unit, and those in the EA group were placed supine on an outpatient bed mat wearing light clothing and diapers. The infants were monitored for $30 \mathrm{~min}$. Video recordings were made at 3-5-min intervals, during which they were spontaneously moving without being excited or crying. Video recordings were recorded $1 \mathrm{~h}$ before or after nursing. The video recordings were made with a camera placed on a tripod directly above the infants. Using the videos, we assessed the behaviour over approximately 1 min during which the infants were alert and awake and showed continuous movements.

Assessment of behaviour in IBC. The behaviours and assessment criteria for the IBC are listed in Table 4. In this study, we have developed a list of observation items based on methods from the Neurological Assessment of the Preterm and Full-term Newborn Infant ${ }^{38}$ (item numbers 9, 16, 17, and 40-44) and the Newborn Individualized Developmental Care and Assessment Program ${ }^{39}$ (item numbers 33-39), which are commonly used in clinical practice to evaluate the behaviour of infants. In addition, the other items were picked up for postures and behaviour that the authors feel were commonly observed in clinical practice.

Items related to posture (item numbers 1 - 17) were judged 'present' when the condition was observed for more than half of the observation time; if not, they were judged 'absent'. This criterion is based on the criteria for evaluating the temporal organization of fidgety movements in the Assessment of General Movements ${ }^{30}$ and the activity in the Neonatal Behavioral Assessment Scale ${ }^{40}$.

Items related to behaviour (item numbers 18-44) were judged 'present' when the behaviour was observed at least twice (uni- or bilaterally); if not, they were judged 'absent'. Seven of the 44 items (item numbers 2, 13, 16, $24,31,32$, and 38 ) were not included in the NA assessments because neonates cannot hold their heads upright or coordinate separate peripheral movements. For consistency, assessments using the IBC were performed by a 


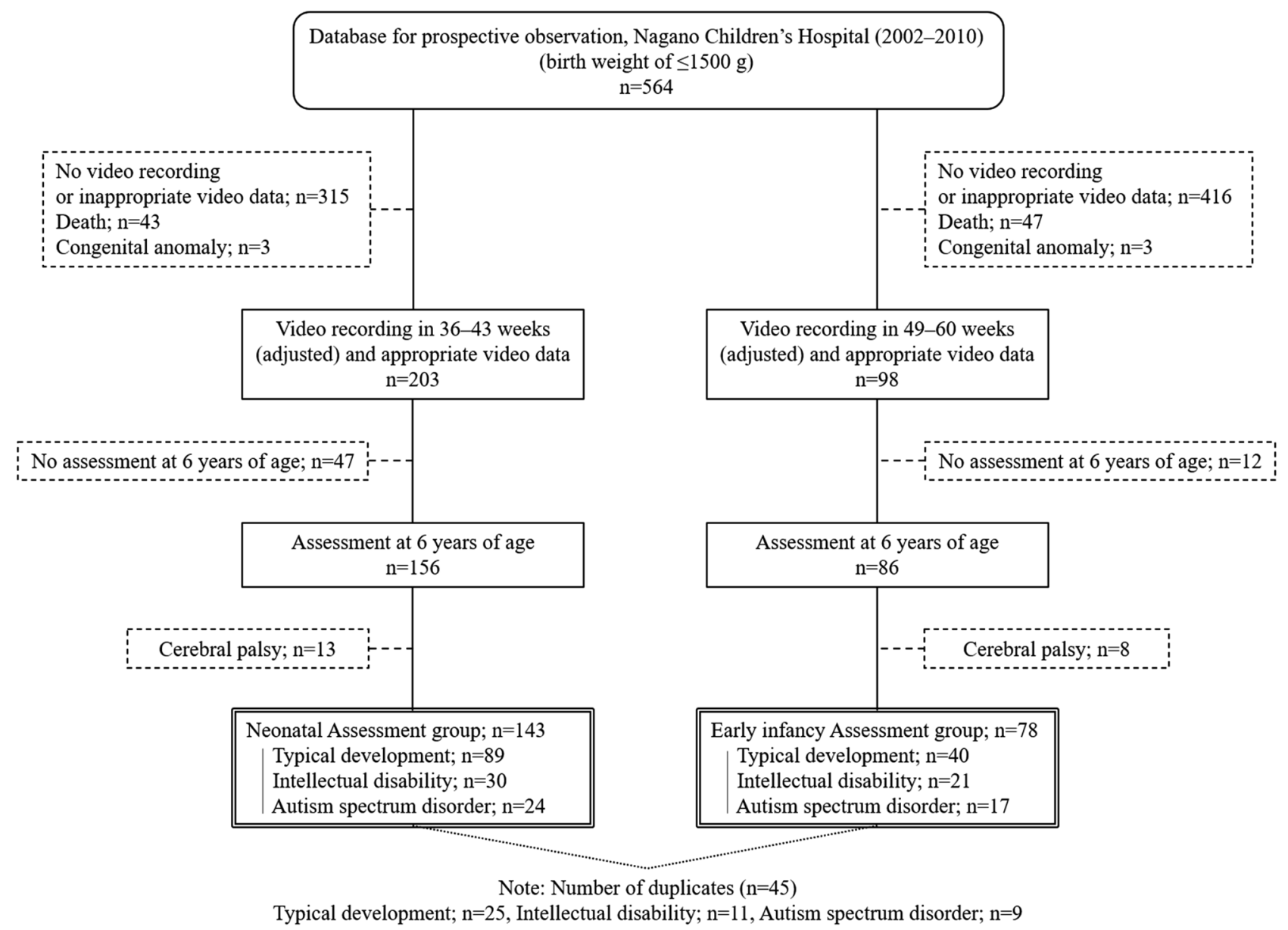

Figure 2. Flow diagram showing study participant enrolment for behavioural assessment during the neonatal and early infancy periods.

single physical therapist (H.K.). Note that the assessor was blinded with regards to the participants' characteristics (sex, birth weight, gestational age, and developmental outcome).

Assessment of developmental outcome at 6 years of age. Developmental outcomes were determined for children aged 6 years. The WISC III was administered at the development outpatient department of Nagano Children's Hospital. The tests were administered by a speech-language-hearing therapist or a clinical psychologist. The WISC is an analytical intelligence test applied to children aged 5 to 16 years, in which verbal IQ, performance IQ, and full-scale IQ are each assessed as a deviation IQ (mean 100, one standard deviation 15) indicating their relative position in the same age group ${ }^{41,42}$. In this study, according to the criteria of the WISC, full-scale IQ of $\geq 80$ was considered normal; 70-79, borderline; and $\leq 69$, delayed. Diagnoses were made by a paediatric psychiatrist using the results of the WISC, brain imaging findings, and the criteria listed in the Diagnostic and Statistical Manual of Mental Disorders (fourth edition). Based on the diagnoses, the children were assigned to the TD, ID, ASD, or CP groups. Children who could not be diagnosed with ASD or CP but who had an IQ of $\geq 80$ were placed in the TD group, and those with an IQ of $\leq 79$ were placed in the ID group.

Statistical analyses. The background characteristics of all groups were compared using the Mann-Whitney $U$ test. We calculated the frequency of observance of each behaviour in each group. Fisher's exact test was used to compare the frequency of behaviours in the ID, ASD, and TD groups. Note that we corrected the p-values using the Benjamini-Hochberg method to account for the effect of false positives due to multiple testing ${ }^{43}$. The False Discovery Rate was set to 0.05 in order to carry out an exploratory test to find the important items. 


\begin{tabular}{|c|c|c|c|}
\hline & No & Item & Assessment criteria \\
\hline \multirow{17}{*}{ Items related posture } & 1 & Neck extension & Maintains neck extension (at least $10^{\circ}$ ); neck at the midline or rotated \\
\hline & $2^{*}$ & Head at the midline & Maintains head at the midline; neck at the midline \\
\hline & 3 & Asymmetrical tonic neck reflex & $\begin{array}{l}\text { Asymmetric tonic neck reflex; upper and lower limbs extended toward } \\
\text { the face, flexed on the opposite side }\end{array}$ \\
\hline & 4 & Trunk extension & Maintains trunk extension (at least $10^{\circ}$ ); trunk at the midline or rotated \\
\hline & 5 & Body co-contraction & Overexerted body; limb and trunk muscles contracted simultaneously \\
\hline & 6 & Elbow flexion & $\begin{array}{l}\text { Elbows maintained in the flexed position (at least } 90^{\circ} \text { ); shoulder rotated } \\
\text { inward }\end{array}$ \\
\hline & 7 & Elbow extension & $\begin{array}{l}\text { Elbows maintained in the extended position (less than } 10^{\circ} \text { ); shoulders } \\
\text { rotated inward }\end{array}$ \\
\hline & 8 & Finger semi-flexion & Fingers incompletely flexed and not grasping \\
\hline & 9 & Thumb adduction & $\begin{array}{l}\text { Thumbs maintained in the adducted position toward the palm (at least } \\
\left.60^{\circ}\right) \text {; other fingers extended }\end{array}$ \\
\hline & 10 & Lower limb crossing & $\begin{array}{l}\text { Hips maintained in the adducted position (at least } 10^{\circ} \text { ); hips in flexed } \\
\text { position }\end{array}$ \\
\hline & 11 & Lower limb abduction & $\begin{array}{l}\text { Hips maintained in the abducted position (at least } 10^{\circ} \text { ); hips in flexed } \\
\text { position }\end{array}$ \\
\hline & 12 & Lower limb flexion and elevation & $\begin{array}{l}\text { Hips and knees maintained in the flexed position (both at least } 60^{\circ} \text { ); feet } \\
\text { off the floor }\end{array}$ \\
\hline & $13^{*}$ & Lower limb extension and elevation & $\begin{array}{l}\text { Maintain the hips in flexed position (at least } 60^{\circ} \text { ) and the knees in } \\
\text { extended position (less than } 10^{\circ} \text { ); feet off the floor }\end{array}$ \\
\hline & 14 & Lower limb extension and ankle dorsiflexion & $\begin{array}{l}\text { Maintain the hips and knees extended and ankles in the dorsiflexed } \\
\text { position (at least } 10^{\circ} \text { ) }\end{array}$ \\
\hline & 15 & Lower limb extension and ankle plantar flexion & $\begin{array}{l}\text { Maintain the hips and knees extended and ankles in the plantar flexed } \\
\text { position (at least } 10^{\circ} \text { ) }\end{array}$ \\
\hline & $16^{*}$ & Toe flexion & Five toes maintained in the flexed position \\
\hline & 17 & Great toe extension & $\begin{array}{l}\text { Great toe maintained in the extended position (at least } 10^{\circ} \text { ); other toes at } \\
\text { the midline or flexed }\end{array}$ \\
\hline \multirow{24}{*}{ Items related behaviour } & 18 & Neck rotation & Neck rotated (at least $10^{\circ}$ ); rotation crosses the midline \\
\hline & 19 & Trunk rotation & Trunk rotated (at least $10^{\circ}$ ); trunk flexed or extended \\
\hline & 20 & Trunk lateral bending & $\begin{array}{l}\text { Trunk bent laterally (at least } 10^{\circ} \text { ); trunk in the flexed or extended posi- } \\
\text { tion }\end{array}$ \\
\hline & 21 & Upper limb flexion and forearm pronation and supination & $\begin{array}{l}\text { Elbow flexed (at least } 60^{\circ} \text { ) and forearm rotated inward or outward (both } \\
\text { at least } 10^{\circ} \text { ) }\end{array}$ \\
\hline & 22 & Upper limb extension and forearm pronation and supination & $\begin{array}{l}\text { Elbow extended (less than } 10^{\circ} \text { ) and forearm rotated inward or outward } \\
\text { (both at least } 10^{\circ} \text { ) }\end{array}$ \\
\hline & 23 & Upper limb swing & $\begin{array}{l}\text { Repeated swinging of the upper limb; repeated raising and lowering with } \\
\text { the upper limb extended }\end{array}$ \\
\hline & $24^{*}$ & Wrist rotation & $\begin{array}{l}\text { Wrist rotated; hand movement in the following order: palmar flexion, } \\
\text { radial flexion, dorsal flexion, and ulnar flexion }\end{array}$ \\
\hline & 25 & Separate finger flexion and extension & Five fingers of the hand flexed and extended separately \\
\hline & 26 & Kicking & Lower limbs repeatedly flexed and extended; lower limbs flexed in turn \\
\hline & 27 & Lower limb flexion and hip internal rotation and external rotation & $\begin{array}{l}\text { Repetitive internal and external rotation of the hips (at least } 10^{\circ} \text { ) with } \\
\text { hips and knees flexed (both at least } 60^{\circ} \text { ) }\end{array}$ \\
\hline & 28 & $\begin{array}{l}\text { Lower limb extension and hip internal rotation and external rotation } \\
\text { dorsiflexion }\end{array}$ & $\begin{array}{l}\text { Repetitive internal and external rotation of the hips (at least } 10^{\circ} \text { ) with } \\
\text { hips and knees extended (both less than } 10^{\circ} \text { ) }\end{array}$ \\
\hline & 29 & Lower limb flexion and ankle dorsiflexion and plantar flexion & $\begin{array}{l}\text { Repetitive dorsiflexion and plantarflexion of ankles (at least } 10^{\circ} \text { ) with } \\
\text { hips and knees flexed (both at least } 60^{\circ} \text { ) }\end{array}$ \\
\hline & 30 & Lower limb extension and ankle dorsiflexion and plantar flexion & $\begin{array}{l}\text { Repetitive dorsiflexion and plantarflexion of ankles (at least } 10^{\circ} \text { ) with } \\
\text { hips and knees extended (both less than } 10^{\circ} \text { ) }\end{array}$ \\
\hline & $31^{*}$ & Ankle inversion and eversion & Repetitive inversion and eversion of ankles \\
\hline & $32^{*}$ & Toe flexion and extension separately & Five toes separately flexed and extended \\
\hline & 33 & Kicking the floor & $\begin{array}{l}\text { In the supine position; knees flexed and the plantar surface of the feet } \\
\text { kicking the floor }\end{array}$ \\
\hline & 34 & Hand to head and ear movement & Hand touching the head or the ear; fingers or palm in contact \\
\hline & 35 & Hand to face movement & Hand touching the face; fingers or palm in contact \\
\hline & 36 & Hand to mouth movement & Hand touching the mouth; fingers or palm in contact \\
\hline & 37 & Hand to hand movement & Both hands touching; fingers or palms in contact \\
\hline & $38^{*}$ & Hand to knee movement & Hand touching the knee; fingers or palm in contact \\
\hline & 39 & Foot to leg movement & $\begin{array}{l}\text { Foot touching the other lower limb; toes or the plantar surface of the } \\
\text { foot in contact }\end{array}$ \\
\hline & 40 & Single limb tremor & Trembling of one limb \\
\hline & 41 & Two or more limb tremor & Trembling of at least two limbs; simultaneous tremors \\
\hline & & & \\
\hline
\end{tabular}




\begin{tabular}{|l|r|l|l|}
\hline & No & Item & Assessment criteria \\
\hline \multirow{2}{*}{42} & Startle response & $\begin{array}{l}\text { Spontaneous Moro reflex; all four limbs simultaneously extended and } \\
\text { outward }\end{array}$ \\
\cline { 2 - 4 } & 43 & Repeat tremor and startle response & Repeated tremors and startled response within $10 \mathrm{~s}$ \\
\cline { 2 - 4 } & 44 & Tremor and startle response with a cry & Tremor and startle response accompanied by crying \\
\hline
\end{tabular}

Table 4. Items of Infant's Behaviour Checklist (IBC) and assessment criteria. ${ }^{\star}$ Assessed only during the early infancy period.

Received: 12 April 2021; Accepted: 15 September 2021

Published online: 29 September 2021

\section{References}

1. Isayama, T. et al. Comparison of mortality and morbidity of very low-birth-weight infants between Canada and Japan. Pediatrics 130, e957-965 (2012).

2. Kusuda, S., Fujimura, M., Uchiyama, A., Totsu, S. \& Matsunami, K. Trends in morbidity and mortality among very-low-birthweight infants from 2003 to 2008 in Japan. Pediatr. Res. 72, 531-538 (2012).

3. Hack, M. et al. Outcomes in young adulthood for very-low-birth-weight infants. N. Engl. J. Med. 346, 149-157 (2002).

4. Hutchinson, E. A. et al. School-age outcomes of extremely preterm or extremely low-birth-weight children. Pediatrics 131, e10531061 (2013).

5. Burd, L., Severud, R., Kerbeshian, J. \& Klug, M. G. Prenatal and perinatal risk factors for autism. J. Perinat. Med. 27, 441-450 (1999).

6. Larsson, H. J. et al. Risk factors for autism: Perinatal factors, parental psychiatric history, and socioeconomic status. Am. J. Epidemiol. 161, 916-925 (2005).

7. Schendel, D. \& Bhasin, T. K. Birth weight and gestational age characteristics of children with autism, including a comparison with other developmental disabilities. Pediatrics 121, 1155-1164 (2008).

8. Williams, K., Helmer, M., Duncan, G. W., Peat, J. K. \& Mellis, C. M. Perinatal and maternal risk factors for autism spectrum disorders in New South Wales, Australia. Child Care Health Dev. 34, 249-256 (2008).

9. Johnson, S. \& Marlow, N. Preterm birth and childhood psychiatric disorders. Pediatr. Res. 69, 11-18 (2011).

10. Prechtl, H. F. Qualitative changes of spontaneous movements in fetus and preterm infants are a marker of neurological dysfunction. Early Hum. Dev. 23, 151-158 (1990).

11. Novak, I. et al. Early, accurate diagnosis and early intervention in cerebral palsy: Advances in diagnosis and treatment. JAMA Pediatr. 171, 897-907 (2017).

12. Morgan, C. et al. The pooled diagnostic accuracy of neuroimaging, general movements, and neurological examination for diagnosing cerebral palsy early in high-risk infants: A case control study. J. Clin. Med. 8, 1879 (2019).

13. Butcher, P. R. et al. The quality of preterm infants' spontaneous movements: An early indicator of intelligence and behaviour at school age. J. Child Psychol. Psychiatry. 50, 920-930 (2009).

14. Bruggink, J. L., Van Braeckel, K. N. \& Bos, A. F. The early motor repertoire of children born preterm is associated with intelligence at school age. Pediatrics 125, e1356-1363 (2010).

15. Phagava, H. et al. General movements in infants with autism spectrum disorders. Georgian Med. News 156, 100-105 (2008).

16. Einspieler, C. et al. Highlighting the first 5 months of life: General movements in infants later diagnosed with autism spectrum disorder or Rett syndrome. Res. Autism Spect. Disord. 8, 286-291 (2014).

17. Zappella, M. et al. What do home videos tell us about early motor and socio-communicative behaviours in children with autistic features during the second year of life-An exploratory study. Early Hum. Dev. 91, 569-575 (2015).

18. Teitelbaum, P., Teitelbaum, O., Nye, J. \& Maurer, R. G. Movement analysis in infancy may be useful for early diagnosis of autism. Proc. Natl. Acad. Sci. USA 95, 13982-13987 (1998).

19. Ohgi, S., Takahashi, T., Nugent, L. K., Arisawa, K. \& Akiyama, T. Neonatal behavioural characteristics and later behavioural problems. Clin. Pediatr. (Phila) 42, 679-686 (2003).

20. Esposito, G., Venuti, P., Maestro, S. \& Muratori, F. An exploration of symmetry in early autism spectrum disorders: Analysis of lying. Brain Dev. 31, 131-138 (2009).

21. Karmel, B. Z. et al. Early medical and behavioural characteristics of NICU infants later classified with ASD. Pediatrics 126, 457-467 (2010).

22. Brisson, J., Warreyn, P., Serres, J., Foussier, S. \& Adrien-Louis, J. Motor anticipation failure in infants with autism: A retrospective analysis of feeding situations. Autism 16, 420-429 (2012).

23. Flanagan, J. E., Landa, R., Bhat, A. \& Bauman, M. Head lag in infants at risk for autism: A preliminary study. Am. J. Occup. Ther. 66, 577-585 (2012).

24. Kihara, H. \& Nakamura, T. Early standard development assessment characteristics in very low-birth-weight infants later classified with autism spectrum disorder. Early Hum. Dev. 91, 357-359 (2015).

25. Heathcock, J. C., Tanner, K., Robson, D., Young, R. \& Lane, A. E. Retrospective analysis of motor development in infants at high and low risk for autism spectrum disorder. Am. J. Occup. Ther. 69, 6905185070 (2015).

26. Gima, H. et al. Early motor signs of autism spectrum disorder in spontaneous position and movement of the head. Exp. Brain Res. 236, 1139-1148 (2018)

27. Kanemaru, N. et al. Specific characteristics of spontaneous movements in preterm infants at term age are associated with developmental delays at age 3 years. Dev. Med. Child Neurol. 55, 713-721 (2013).

28. Kanemaru, N. et al. Jerky spontaneous movements at term age in preterm infants who later developed cerebral palsy. Early Hum. Dev. 90, 387-392 (2014).

29. Gima, H. et al. Assessment of fidgety movements in low-birth-weight infants based on visual observation and computer-based analysis [in Japanese, English abstract]. Phys. Ther. Jpn. 44, 115-123 (2017).

30. Einspieler, C., Prechtl, H. F., Bos, A. F., Ferrari, F. \& Cioni, G. Prechtl's Method on the Qualitative Assessment of General Movements in Preterm, Term And young Infants (Mac Keith Press, 2004).

31. Hadders-Algra, M. Neural substrate and clinical significance of general movements: An update. Dev. Med. Child Neurol. 60, 39-46 (2018).

32. Prechtl, H. F. State of the art of a new functional assessment of the young nervous system: An early predictor of cerebral palsy. Early Hum. Dev. 50, 1-11 (1997).

33. Prechtl, H. F. et al. An early marker for neurological deficits after perinatal brain lesions. Lancet 349, 1361-1363 (1997). 
34. Wilson, R. B., Vangala, S., Elashoff, D., Safari, T. \& Smith, B. A. Using wearable sensor technology to measure motion complexity in infants at high familial risk for autism spectrum disorder. Sensors (Basel). 21, 616 (2021).

35. Gima, H., Shimatani, K., Nakano, H., Watanabe, H. \& Taga, G. Evaluation of fidgety movements of infants based on gestalt perception reflects differences in limb movement trajectory curvature. Phys. Ther. 99, 701-710 (2019).

36. Dawson, G. et al. Randomized, controlled trial of an intervention for toddlers with autism: The Early Start Denver Model. Pediatrics 125, 17-23 (2010)

37. Landa, R. J., Holman, K. C., O’Neill, A. H. \& Stuart, E. A. Intervention targeting development of socially synchronous engagement in toddlers with autism spectrum disorder: A randomized controlled trial. J. Child Psychol. Psychiatry 52, 13-21 (2011).

38. Dubowitz, L. M. et al. The Neurological Assessment of the Preterm and Full-term Newborn Infant 2nd edn. (Mac Keith Press, 1999).

39. Als, H. Newborn Individualized Developmental Care and Assessment Program (NIDCAP): New frontier for neonatal and perinatal medicine. J. Neonatal. Perinat. Med. 2, 135-147 (2009).

40. Brazelton, T. B. \& Nugent, J. K. Neonatal Behavioral Assessment Scale 4th edn. (Mac Keith Press, 2011).

41. Wechsler, D. Wechsler Intelligence Scale for Children 3rd edn. (Psychological Corporation, 1991).

42. Wechsler, D. Wechsler Intelligence Scale for Children 4th edn. (Psychological Corporation, 2003).

43. Benjamini, Y. \& Hochberg, Y. Controlling the false discovery rate: A practical and powerful approach to multiple testing. J. R. Stat. Soc. 57, 289-300 (1995).

\section{Acknowledgements}

We thank Junji Nakano of Chuo University for advice on the statistical analysis of this study.

\section{Author contributions}

H.K. conceptualized and designed the study, collected all data, carried out the initial analyses, drafted the initial manuscript, and approved the final manuscript as submitted. H.N. conceptualized and designed the study, made substantial contributions to the data analyses, reviewed the manuscript, and approved the final manuscript as submitted. T.N. conceptualized and designed the study, coordinated and supervised data collection, reviewed and revised the manuscript, and approved the final manuscript as submitted. H.G. supervised the data analyses, critically reviewed and revised the manuscript, and approved the final manuscript as submitted. All authors approved the final manuscript as submitted and agree to be accountable for all aspects of the work.

\section{Competing interests}

The authors declare no competing interests.

\section{Additional information}

Supplementary Information The online version contains supplementary material available at https://doi.org/ 10.1038/s41598-021-98884-y.

Correspondence and requests for materials should be addressed to H.G.

Reprints and permissions information is available at www.nature.com/reprints.

Publisher's note Springer Nature remains neutral with regard to jurisdictional claims in published maps and institutional affiliations.

Open Access This article is licensed under a Creative Commons Attribution 4.0 International License, which permits use, sharing, adaptation, distribution and reproduction in any medium or format, as long as you give appropriate credit to the original author(s) and the source, provide a link to the Creative Commons licence, and indicate if changes were made. The images or other third party material in this article are included in the article's Creative Commons licence, unless indicated otherwise in a credit line to the material. If material is not included in the article's Creative Commons licence and your intended use is not permitted by statutory regulation or exceeds the permitted use, you will need to obtain permission directly from the copyright holder. To view a copy of this licence, visit http://creativecommons.org/licenses/by/4.0/.

(C) The Author(s) 2021 